\title{
Extremes of independent chi-square random vectors
}

\author{
Enkelejd Hashorva - Zakhar Kabluchko• \\ Achim Wübker
}

Received: 23 June 2010 / Revised: 23 June 2010 /

Accepted: 18 October 2010 / Published online: 12 November 2010

(C) Springer Science+Business Media, LLC 2010

\begin{abstract}
We prove that the componentwise maximum of an i.i.d. triangular array of chi-square random vectors converges in distribution, under appropriate assumptions on the dependence within the vectors and after normalization, to the max-stable Hüsler-Reiss distribution. As a by-product we derive a conditional limit result.
\end{abstract}

Keywords Extremes $\cdot$ Multivariate chi-square distribution $\cdot$ Gaussian random vector · Hüsler-Reiss distribution · Max-stable distribution · Conditional limit result

AMS 2000 Subject Classifications Primary 60F05; Secondary 60 G70

\footnotetext{
E. Hashorva $(\varangle)$

Department of Mathematical Statistics and Actuarial Science, University of Bern, Sidlerstrasse 5, 3012, Bern, Switzerland

e-mail: hashorva@stat.unibe.ch

\section{E. Hashorva}

Faculty of Business and Economics, University of Lausanne, Extranef, UNIL-Dorigny, 1015, Lausanne, Switzerland
}

\section{Z. Kabluchko}

Institute of Stochastics, Ulm University, Helmholtzstr. 18, 89069, Ulm, Germany

e-mail: zakhar.kabluchko@uni-ulm.de

\section{A. Wübker}

University of Osnabrück, Albrechtstr. 28a, 49076, Osnabrück, Germany

e-mail: awuebker@uni-osnabrueck.de 


\section{Introduction and main result}

It is well-known that if $X^{(1)}, X^{(2)}, \ldots$ are independent standard Gaussian random variables, then the maximum $M_{n}:=\max _{1 \leq j \leq n} X^{(j)}$ satisfies

$$
\lim _{n \rightarrow \infty} \mathbb{P}\left\{\sqrt{2 \ln n}\left(M_{n}-\left(\sqrt{2 \ln n}-\frac{\ln (4 \pi \ln n)}{2 \sqrt{2 \ln n}}\right)\right) \leq t\right\}=e^{-e^{-t}}, \quad t \in \mathbb{R} .
$$

The following multivariate version of this result has been obtained by Hüsler and Reiss (1989). For every $n \geq 1$ let $\boldsymbol{X}_{n}=\left(X_{n 1}, \ldots, X_{n d}\right)^{\top}$ be a $d$-variate Gaussian random vector with zero-mean, unit-variance margins and a covariance matrix $\Sigma_{n}$. Throughout, ${ }^{\top}$ stands for the transpose sign. Let $\boldsymbol{X}_{n}^{(1)}, \ldots, \boldsymbol{X}_{n}^{(n)}$, where $\boldsymbol{X}_{n}^{(j)}=\left(X_{n 1}^{(j)}, \ldots, X_{n d}^{(j)}\right)^{\top}, 1 \leq j \leq n$, be independent copies of the random vector $\boldsymbol{X}_{n}$. Denote by $\boldsymbol{M}_{n}=\left(M_{n 1}, \ldots, M_{n d}\right)^{\top}, n \geq 1$, the $d$-variate vector of the componentwise sample maxima given by

$$
M_{n i}:=\max _{1 \leq j \leq n} X_{n i}^{(j)}, \quad 1 \leq i \leq d .
$$

Assuming that the sequence of covariance matrices $\Sigma_{n}, n \geq 1$, satisfies

$$
\lim _{n \rightarrow \infty} 4 \ln n \cdot\left(\mathbf{1 1}^{\top}-\Sigma_{n}\right)=\Lambda \in[0, \infty)^{d \times d}, \quad \mathbf{1}=(1, \ldots, 1)^{\top} \in \mathbb{R}^{d},
$$

Hüsler and Reiss (1989) have shown that we have the following convergence in distribution

$$
\sqrt{2 \ln n}\left(\boldsymbol{M}_{n}-\left(\sqrt{2 \ln n}-\frac{\ln (4 \pi \ln n)}{2 \sqrt{2 \ln n}}\right) \mathbf{1}\right) \stackrel{d}{\rightarrow} \mathcal{M}_{\Lambda}, \quad n \rightarrow \infty .
$$

Here, $\mathcal{M}_{\Lambda}$ is a $d$-variate vector having the so-called Hüsler-Reiss distribution, a multivariate max-stable distribution with Gumbel margins; see Hüsler and Reiss (1989) and Falk et al. (2004, Example 4.1.4). For extensions and related results, see Brown and Resnick (1977), Hashorva (2008), Kabluchko et al. (2009) and Kabluchko (2010).

Our aim is to prove an analogue of the Hüsler-Reiss result for $\chi^{2}$-random vectors. Recall that a random variable $\xi$ is said to have a $\chi^{2}$-distribution with $m \in \mathbb{N}$ degrees of freedom, if it can be represented as $\xi \stackrel{d}{=} X_{1}^{2}+\ldots+X_{m}^{2}$, where $X_{1}, \ldots, X_{m}$ are independent standard Gaussian random variables. It is well-known that the $\chi^{2}$ distribution, being a special case of the gamma distribution, is in the max-domain of attraction of the Gumbel distribution; see, e.g., Embrechts et al. (1997, p. 156). More precisely, let $\xi^{(1)}, \xi^{(2)}, \ldots$ be independent $\chi^{2}$-random variables with $m$ degrees of freedom and define

$$
b_{n}=2 \ln n+(m-2) \ln \ln n-2 \ln \Gamma(m / 2) .
$$


It is easy to check that $\mathbb{P}\left\{\xi>b_{n}+2 t\right\} \sim e^{-t} / n$ as $n \rightarrow \infty$. Hence, the maximum $M_{n}:=\max _{1 \leq j \leq n} \xi^{(j)}$ satisfies

$$
\lim _{n \rightarrow \infty} \mathbb{P}\left\{M_{n} \leq b_{n}+2 t\right\}=e^{-e^{-t}}, \quad t \in \mathbb{R} .
$$

Our aim is to prove a multidimensional version of Eq. 1.5. In the case $m=1$, our result will essentially reduce to that of Hüsler-Reiss. For every $n \geq 1$ let $\boldsymbol{\xi}_{n}=$ $\left(\xi_{n 1}, \ldots, \xi_{n d}\right)^{\top}$ be a $d$-variate $\chi^{2}$-random vector with $m$ degrees of freedom whose components are given by

$$
\xi_{n i}=X_{n i ; 1}^{2}+\ldots+X_{n i ; m}^{2}, \quad 1 \leq i \leq d, \quad n \geq 1
$$

where $\boldsymbol{X}_{n ; j}=\left(X_{n 1 ; j}, \ldots, X_{n d ; j}\right)^{\top}, 1 \leq j \leq m$, are $m$ independent copies of a zero-mean, unit-variance $d$-variate Gaussian random vector $\boldsymbol{X}_{n}=\left(X_{n 1}, \ldots, X_{n d}\right)^{\top}$ with covariance matrix $\Sigma_{n}$, as defined above.

Note in passing that for every $1 \leq i \leq d, n \geq 1$, the margin $\xi_{n i}$ has $\chi^{2}$-distribution with $m$ degrees of freedom, and furthermore $\xi_{n 1}, \ldots, \xi_{n d}$ are in general dependent random variables. For every $n \geq 1$ let $\xi_{n}^{(1)}, \ldots, \xi_{n}^{(n)}$ be independent copies of the random vector $\xi_{n}$; we write $\xi_{n}^{(j)}=\left(\xi_{n 1}^{(j)}, \ldots, \xi_{n d}^{(j)}\right)^{\top}, 1 \leq j \leq n$. Let $\boldsymbol{M}_{n}=$ $\left(M_{n 1}, \ldots, M_{n d}\right)^{\top}, n \geq 1$, be the componentwise sample maxima given by

$$
M_{n i}:=\max _{1 \leq j \leq n} \xi_{n i}^{(j)}, \quad 1 \leq i \leq d .
$$

Our main result describing the asymptotic distribution of $\boldsymbol{M}_{\boldsymbol{n}}$ reads as follows.

Theorem 1.1 Assume that the sequence of covariance matrices $\Sigma_{n}, n \geq 1$, satisfies Eq. 1.2 and let the normalizing constants $b_{n}, n \geq 1$, be defined by Eq. 1.4. Then, we have the following convergence in distribution

$$
\frac{1}{2}\left(M_{n}-b_{n} \mathbf{1}\right) \stackrel{d}{\rightarrow} \mathcal{M}_{\Lambda}, \quad n \rightarrow \infty
$$

Several authors have studied large deviations for the maxima of continuous-time $\chi^{2}$-square processes; see Albin (1990) and the references therein. In our proof of Theorem 1.1 we will rely on some ideas of Hüsler and Reiss (1989) and Albin (1990).

\section{Discussion}

The crucial role in the triangular array scheme considered by Hüsler and Reiss (1989) is played by the limit condition (Eq. 1.2) which specifies the rate of convergence of the components of the covariance matrix $\Sigma_{n}$ to 1 . In fact, it is known that if the off-diagonal elements of $\Sigma_{n}$ remain bounded away from 1 as $n \rightarrow \infty$, then the components of the maximum $\boldsymbol{M}_{n}$ become independent in the limit. In this case, 
the off-diagonal elements of the matrix $\Lambda$ in Eq. 1.2 are equal to $+\infty$. On the other hand, if the matrix $\Lambda$ is equal to zero, then the components of the limiting distribution are a.s. equal. The result of Hüsler and Reiss interpolates between these boundary cases. The space of Hüsler-Reiss distributions may be "compactified" by allowing the elements of the matrix $\Lambda$ to be infinite (for a rigorous treatment, see, e.g., the notion of a negative defined kernel in the extended sense in Kabluchko 2010).

Our result shows that also the maxima of triangular arrays of i.i.d. $\chi^{2}$-random vectors are attracted by the Hüsler-Reiss distributions, thus extending the applicability of the Hüsler-Reiss distributions beyond the Gaussian setup.

Due to the importance of conditional limit theorems and conditional extreme value models (see Heffernan and Resnick 2007; Das and Resnick 2010) we provide a conditional limit result for $\chi^{2}$-distributions. We focus for simplicity on the 2-dimensional setup considering $\xi_{n}=\left(\xi_{n 1}, \xi_{n 2}\right)^{\top}, n \geq 1$, as in Eq. 1.6, where the underlying covariance matrix $\Sigma_{n}$ has off-diagonal elements equal $\rho_{n} \in(-1,1), n \geq 1$, and its main diagonal consists of 1's.

Theorem 2.1 Let $b_{n}, n \geq 1$, be a sequence of constants such that $\lim _{n \rightarrow \infty} b_{n}=\infty$ and $\lim _{n \rightarrow \infty} 2 b_{n}\left(1-\rho_{n}\right)=\lambda \in[0, \infty)$. Then for any $x \in \mathbb{R}$,

$$
\lim _{n \rightarrow \infty} \mathbb{P}\left\{\xi_{n 2}>b_{n}+2 x \mid \xi_{n 1}>b_{n}\right\}=\mathbb{P}\left\{\sqrt{\lambda} Z-\frac{\lambda}{2}+S>x\right\},
$$

where $Z$ is a standard normal variable and $S$ is a unit-mean exponential random variable independent of $Z$.

\section{Proofs}

Proof of Theorem 1.1 First we introduce some notation. Given a vector $\boldsymbol{x}=$ $\left(x_{1}, \ldots, x_{d}\right)^{\top} \in \mathbb{R}^{d}$ we define $\boldsymbol{x}_{K}:=\left(x_{2}, \ldots, x_{d}\right)^{\top}$ with $K=\{2, \ldots, d\}$. If $A=\left(a_{i j}\right) \in \mathbb{R}^{d \times d}$ is a matrix, then we define $A_{11}=a_{11}, A_{1 K}:=\left(a_{12}, \ldots, a_{1 d}\right)$, $A_{K 1}:=\left(a_{21}, \ldots, a_{d 1}\right)^{\top}$, and $A_{K K}:=\left(a_{i j}\right)_{i, j \in K}$. Given $\boldsymbol{x}, \boldsymbol{y} \in \mathbb{R}^{d}$ write

$$
\begin{aligned}
\boldsymbol{x} & >\boldsymbol{y}, \text { if } x_{i}>y_{i} \text { for every } 1 \leq i \leq d, \\
\boldsymbol{x}+\boldsymbol{y} & :=\left(x_{1}+y_{1}, \ldots, x_{d}+y_{d}\right)^{\top}, \quad c \boldsymbol{x}:=\left(c x_{1}, \ldots, c x_{d}\right)^{\top}, \quad c \in \mathbb{R}, \\
\boldsymbol{x} \boldsymbol{y} & :=\left(x_{1} y_{1}, \ldots, x_{d} y_{d}\right)^{\top}, \quad \boldsymbol{x}^{2}:=\boldsymbol{x} \boldsymbol{x} .
\end{aligned}
$$

In view of Lemma 4.1.3 in Falk et al. (2004), the proof of the theorem follows if we show that

$$
\begin{aligned}
& \lim _{n \rightarrow \infty} n \mathbb{P}\left\{\boldsymbol{\xi}_{n}>b_{n} \mathbf{1}+2 \boldsymbol{x}\right\} \\
& =\int_{x_{1}}^{\infty} \mathbb{P}\left\{\boldsymbol{W}>\boldsymbol{x}_{K}-s \mathbf{1}_{K}+\frac{1}{2} \Lambda_{K 1}\right\} e^{-s} d s, \quad x \in \mathbb{R}^{d}
\end{aligned}
$$


where $\boldsymbol{W}$ is a $(d-1)$-variate mean-zero Gaussian random vector with covariance matrix $\Gamma \in \mathbb{R}^{(d-1) \times(d-1)}$ defined by

$$
\Gamma:=\left(\mathbf{1}_{K} \Lambda_{1 K}+\Lambda_{K 1} \mathbf{1}_{K}^{\top}-\Lambda_{K K}\right) / 2
$$

Indeed, an application of Eq. 3.1 to the subvectors of $\boldsymbol{\xi}_{n}$ verifies the conditions of Lemma 4.1.3 in Falk et al. (2004).

Since $\Sigma_{n}$ is a positive definite matrix, we can find a matrix $A_{n}$ such that $\Sigma_{n}=$ $A_{n} A_{n}^{\top}$. Let $\boldsymbol{Z}_{1}, \ldots, \boldsymbol{Z}_{m}$, where $\boldsymbol{Z}_{l}:=\left(Z_{1 ; l}, \ldots, Z_{d ; l}\right)^{\top}, 1 \leq l \leq m$, be $m$ independent standard Gaussian random vectors in $\mathbb{R}^{d}$ whose covariance matrix is equal to the identity matrix in $\mathbb{R}^{d}$. Further, let $B_{n} \in \mathbb{I}^{(d-1) \times(d-1)}$ be a square matrix such that

$$
B_{n} B_{n}^{\top}=\left(\Sigma_{n}\right)_{K K}-\sigma_{n} \sigma_{n}^{\top}, \quad \sigma_{n}:=\left(\Sigma_{n}\right)_{K 1} .
$$

Note that the matrix $\left(\Sigma_{n}\right)_{K K}-\sigma_{n} \boldsymbol{\sigma}_{n}^{\top}$, being the Schur complement of $\left(\Sigma_{n}\right)_{11}$, is positive definite. It follows that for any $n \geq 1$ we have the joint stochastic representation

$$
\xi_{n 1} \stackrel{d}{=} \sum_{l=1}^{m} Z_{1 ; l}^{2}, \quad\left(\xi_{n 2}, \ldots, \xi_{n d}\right)^{\top} \stackrel{d}{=} \sum_{l=1}^{m}\left(B_{n} Z_{K ; l}+Z_{1 ; l} \boldsymbol{\sigma}_{\boldsymbol{n}}\right)^{2},
$$

where we set $Z_{K ; l}:=\left(Z_{2 ; l}, \ldots, Z_{d ; l}\right)^{\top}$. Consequently, since $Z_{1 ; l}$ is independent of $\boldsymbol{Z}_{K ; l}$ for any $1 \leq l \leq m$, for every $\boldsymbol{x} \in \mathbb{R}^{d}$ and $\varepsilon>0$ we obtain

$$
\begin{aligned}
\mathbb{P}\left\{\boldsymbol{\xi}_{n}>b_{n} \mathbf{1}+2 \boldsymbol{x}\right\} & \left\{\sum_{l=1}^{m} Z_{1 ; l}^{2}>b_{n}+2 x_{1}, \sum_{l=1}^{m}\left(B_{n} \boldsymbol{Z}_{K ; l}+Z_{1 ; l} \boldsymbol{\sigma}_{\boldsymbol{n}}\right)^{2}>b_{n} \mathbf{1}_{K}+2 \boldsymbol{x}_{K}\right\} \\
\leq & \mathbb{P}\left\{\sum_{l=1}^{m} Z_{1 ; l}^{2}>b_{n}+2 x_{1}, \sum_{l=1}^{m}\left(B_{n} \boldsymbol{Z}_{K ; l}+Z_{1 ; l} \boldsymbol{\sigma}_{n}\right)^{2}>b_{n} \mathbf{1}_{K}\right. \\
& \left.+2 \boldsymbol{x}_{K}, \sum_{l=1}^{m}\left(B_{n} \boldsymbol{Z}_{K ; l}\right)^{2} \leq \varepsilon \mathbf{1}_{K}\right\} \\
+ & \mathbb{P}\left\{\sum_{l=1}^{m} Z_{1 ; l}^{2}>b_{n}+2 x_{1}\right\}\left(1-\mathbb{P}\left\{\sum_{l=1}^{m}\left(B_{n} \boldsymbol{Z}_{K ; l}\right)^{2} \leq \varepsilon \mathbf{1}_{K}\right\}\right) \\
\leq & \mathbb{P}\left\{\sum_{l=1}^{m} Z_{1 ; l}^{2}>b_{n}+2 x_{1}, 2 \sum_{l=1}^{m} Z_{1 ; l}\left(B_{n} \boldsymbol{Z}_{K ; l}\right) \boldsymbol{\sigma}_{n}\right. \\
& \left.+\sum_{l=1}^{m} Z_{1 ; l}^{2} \boldsymbol{\sigma}_{n}^{2}>2 \boldsymbol{x}_{K}-\varepsilon \mathbf{1}_{K}+b_{n} \mathbf{1}_{K}\right\} \\
+ & \mathbb{P}\left\{\sum_{l=1}^{m} Z_{1 ; l}^{2}>b_{n}+2 x_{1}\right\}\left(1-\mathbb{P}\left\{\sum_{l=1}^{m}\left(B_{n} \boldsymbol{Z}_{K ; l}\right)^{2} \leq \varepsilon \mathbf{1}_{K}\right\}\right)
\end{aligned}
$$


Recall from Eq. 3.3 that $\sigma_{n}:=\left(\Sigma_{n}\right)_{K 1}$. Condition 1.2 implies that

$$
\lim _{n \rightarrow \infty} \boldsymbol{\sigma}_{n}=\mathbf{1}_{K}, \quad \lim _{n \rightarrow \infty} b_{n}\left[\mathbf{1}_{K}-\boldsymbol{\sigma}_{n}^{2}\right]=\lim _{n \rightarrow \infty} 2 b_{n}\left[\mathbf{1}_{K}-\boldsymbol{\sigma}_{n}\right]=\Lambda_{K 1}
$$

Also, it follows from Eq. 1.2 and the definition of $B_{n}$ in Eq. 3.3 that

$$
\lim _{n \rightarrow \infty} b_{n} B_{n} B_{n}^{\top}=\left(\mathbf{1}_{K} \Lambda_{1 K}+\Lambda_{K 1} \mathbf{1}_{K}^{\top}-\Lambda_{K K}\right) / 2=\Gamma
$$

Let $F$ be the $\chi^{2}$-distribution function with $m$ degrees of freedom. By the choice of the sequence $b_{n}$ in Eq. 1.4 ,

$$
\lim _{n \rightarrow \infty} n\left(1-F\left(b_{n}+2 s\right)\right)=e^{-s}, \quad s \in \mathbb{R}
$$

It follows from Eq. 3.6 that the elements of the matrix $B_{n}$ converge to 0 as $n \rightarrow \infty$. Hence,

$$
\lim _{n \rightarrow \infty} \mathbb{P}\left\{\sum_{l=1}^{m} Z_{1 ; l}^{2}>b_{n}+2 x_{1}\right\}\left(1-\mathbb{P}\left\{\sum_{l=1}^{m}\left(B_{n} \boldsymbol{Z}_{K ; l}\right)^{2} \leq \varepsilon \mathbf{1}_{K}\right\}\right)=e^{-x_{1}} \cdot 0=0
$$

Consequently, we have

$$
\begin{aligned}
\limsup _{n \rightarrow \infty} \psi_{n}(\boldsymbol{x}) & \leq \liminf _{n \rightarrow \infty} n \mathbb{P}\left\{\boldsymbol{\xi}_{n}>b_{n} \mathbf{1}+2 \boldsymbol{x}\right\} \leq \limsup _{n \rightarrow \infty} n \mathbb{P}\left\{\boldsymbol{\xi}_{n}>b_{n} \mathbf{1}+2 \boldsymbol{x}\right\} \\
& \leq \liminf _{n \rightarrow \infty} \psi_{n}(\boldsymbol{x}-(\varepsilon / 2) \mathbf{1})
\end{aligned}
$$

where

$$
\begin{aligned}
\psi_{n}(\boldsymbol{x}):=n \mathbb{P}\{ & \sum_{l=1}^{m} Z_{1 ; l}^{2}>b_{n}+2 x_{1}, 2 \sum_{l=1}^{m} Z_{1 ; l}\left(B_{n} \boldsymbol{Z}_{K ; l}\right) \boldsymbol{\sigma}_{n} \\
& \left.+\sum_{l=1}^{m} Z_{1 ; l}^{2} \boldsymbol{\sigma}_{n}^{2}>b_{n} \mathbf{1}_{K}+2 \boldsymbol{x}_{K}\right\}
\end{aligned}
$$

In the rest of the proof we compute $\lim _{n \rightarrow \infty} \psi_{n}(\boldsymbol{x})$. The following stochastic representation is crucial:

$$
\begin{gathered}
2 \sum_{l=1}^{m} Z_{1 ; l}\left(B_{n} \boldsymbol{Z}_{K ; l}\right) \boldsymbol{\sigma}_{n}+\sum_{l=1}^{m} Z_{1 ; l}^{2} \boldsymbol{\sigma}_{n}^{2} \stackrel{d}{=} 2\left[\sqrt{\sum_{l=1}^{m} Z_{1 ; l}^{2}}\right]\left(B_{n} \boldsymbol{Z}_{K ; 1}\right) \boldsymbol{\sigma}_{n}+\left[\sum_{l=1}^{m} Z_{1 ; l}^{2}\right] \boldsymbol{\sigma}_{n}^{2} \\
\stackrel{d}{=} 2 \sqrt{\xi}\left(B_{n} \boldsymbol{Z}_{K ; 1}\right) \boldsymbol{\sigma}_{n}+\xi \boldsymbol{\sigma}_{n}^{2}
\end{gathered}
$$


where the $\chi^{2}$-variable $\xi:=\sum_{l=1}^{m} Z_{1 ; l}^{2}$ is independent of $\boldsymbol{Z}_{K ; 1}$. Recall that the distribution function of $\xi$ is denoted by $F$ and write $F_{n}(s)=n\left(1-F\left(b_{n}+2 s\right)\right)$ for $n>1, s \in \mathbb{R}$. By conditioning on $\xi=s$ we obtain

$$
\begin{aligned}
& \psi_{n}(\boldsymbol{x}) \\
& =n \int_{b_{n}+2 x_{1}}^{\infty} \mathbb{P}\left\{2 \sqrt{\xi}\left(B_{n} \boldsymbol{Z}_{K ; 1}\right) \boldsymbol{\sigma}_{n}+\xi \boldsymbol{\sigma}_{n}^{2}>b_{n} \mathbf{1}_{K}+2 \boldsymbol{x}_{K} \mid \xi=s\right\} d F(s) \\
& =-\int_{x_{1}}^{\infty} \mathbb{P}\left\{2 \sqrt{b_{n}+2 s}\left(B_{n} \boldsymbol{Z}_{K ; 1}\right) \boldsymbol{\sigma}_{n}+\left(b_{n}+2 s\right) \boldsymbol{\sigma}_{n}^{2}>b_{n} \mathbf{1}_{K}+2 \boldsymbol{x}_{K}\right\} d F_{n}(s) \\
& =-\int_{x_{1}}^{\infty} \mathbb{P}\left\{\sqrt{b_{n}+2 s}\left(B_{n} \boldsymbol{Z}_{K ; 1}\right) \boldsymbol{\sigma}_{n}>\boldsymbol{x}_{K}-s \boldsymbol{\sigma}_{n}^{2}+b_{n}\left[\mathbf{1}_{K}-\boldsymbol{\sigma}_{n}^{2}\right] / 2\right\} d F_{n}(s) \\
& =:-\int_{x_{1}}^{\infty} q_{n}(s) d F_{n}(s) .
\end{aligned}
$$

It follows from Eqs. 3.5-3.7 that for any sequence of constants $s_{n}, n \geq 1$, such that $\lim _{n \rightarrow \infty} s_{n}=s \in \mathbb{R}$, we have

$$
\lim _{n \rightarrow \infty} q_{n}\left(s_{n}\right)=\mathbb{P}\left\{\boldsymbol{W}>\boldsymbol{x}_{K}-s \mathbf{1}_{K}+\Lambda_{K 1} / 2\right\} \quad \text { and } \quad \lim _{n \rightarrow \infty} F_{n}(s)=e^{-s} .
$$

Also, we have $q_{n}(s) \leq 1$ for every $n \geq 1, s \in \mathbb{R}$. Applying Lemma 4.4 of Hashorva (2006) (or Theorem 3.27 of Kallenberg 1997) we obtain

$$
\left.\lim _{n \rightarrow \infty} \psi_{n}(\boldsymbol{x})=\int_{x_{1}}^{\infty} \mathbb{P}\left\{\boldsymbol{W}>\boldsymbol{x}_{K}-s \mathbf{1}_{K}+\frac{1}{2} \Lambda_{K}\right\}\right\} e^{-s} d s .
$$

By the arbitrariness of $\varepsilon$ in Eq. 3.8, we obtain Eq. 3.1. Restricting Eq. 3.1 to the subvectors of $\xi_{n}$ and applying Lemma 4.1 .3 of Falk et al. (2004) completes the proof.

Proof of Theorem 2.1 In view of Eq. 3.7, we need to show that for every sequence $s_{n}, n \geq 1$, such that $\lim _{n \rightarrow \infty} s_{n}=s$, where $s \geq 0$, we have

$$
\lim _{n \rightarrow \infty} \mathbb{P}\left\{\xi_{n 2}>b_{n}+2 x \mid \xi_{n 1}=b_{n}+2 s_{n}\right\}=\mathbb{P}\left\{\sqrt{\lambda} Z-\frac{\lambda}{2}+s>x\right\} .
$$

Here, $Z$ is a standard Gaussian random variable. We have a joint stochastic representation $\xi_{n 1} \stackrel{d}{=} \sum_{l=1}^{m} Z_{1 ; l}^{2}$ and $\xi_{n 2} \stackrel{d}{=} \sum_{l=1}^{m}\left(\rho_{n} Z_{1 ; l}+\sqrt{1-\rho_{n}^{2}} Z_{2 ; l}\right)^{2}$, where we use the same notation as in the proof of Theorem 1.1. Utilizing Eq. 3.4, the claim (Eq. 3.11) follows if we show that for every $x \in \mathbb{R}$,

$$
\begin{aligned}
& \lim _{n \rightarrow \infty} \mathbb{P}\left\{2 \rho_{n} \sqrt{1-\rho_{n}^{2}} \sum_{l=1}^{m} Z_{1 ; l} Z_{2 ; l}+\rho_{n}^{2} \sum_{l=1}^{m} Z_{1 ; l}^{2}>b_{n}+2 x \mid \sum_{l=1}^{m} Z_{1 ; l}^{2}=b_{n}+2 s_{n}\right\} \\
& =\mathbb{P}\left\{\sqrt{\lambda} Z-\frac{\lambda}{2}+s>x\right\},
\end{aligned}
$$


By assumption, $\lim _{n \rightarrow \infty} \rho_{n}=1$, hence with the same arguments as in Eq. 3.9 the limit on the left-hand side can be written as

$$
\begin{aligned}
& \lim _{n \rightarrow \infty} \mathbb{P}\left\{2 \rho_{n} \sqrt{\left(b_{n}+2 s_{n}\right)\left(1-\rho_{n}^{2}\right)} Z+2 \rho_{n}^{2} s_{n}-b_{n}\left(1-\rho_{n}^{2}\right)>2 x\right\} \\
& \quad=\mathbb{P}\left\{\sqrt{\lambda} Z-\frac{\lambda}{2}+s>x\right\},
\end{aligned}
$$

and thus Eq. 3.11 follows since $\lim _{n \rightarrow \infty} b_{n}\left(1-\rho_{n}^{2}\right)=\lambda$ by assumption.

\section{References}

Albin, P.: On extremal theory for stationary processes. Ann. Probab. 18(1), 92-128 (1990)

Brown, B.M., Resnick, S.I.: Extreme values of independent stochastic processes. J. Appl. Probab. 14, $732-739$ (1977)

Das, B., Resnick, S.I.: Detecting a conditional extreme value model. To appear in Extremes. Preprint available at http://arxiv.org/abs/0902.2996 (2010)

Embrechts, P., Klüppelberg, C., Mikosch, T.: Modelling Extremal Events for Insurance and Finance. Springer, Berlin (1997)

Falk, M., Hüsler, J., Reiss, R.-D.: Laws of small numbers: extremes and rare events. In: DMV Seminar, vol. 23, 2nd edn. Birkhäuser, Basel (2004)

Hashorva, E.: Gaussian approximation of conditional elliptical random vectors. Stoch. Models 22, 441-457 (2006)

Hashorva, E.: Extremes of weighted Dirichlet arrays. Extremes 11(4), 393-420 (2008)

Heffernan, J.E., Resnick, S.I.: Limit laws for random vectors with an extreme component. Ann. Appl. Probab. 17, 537-571 (2007)

Hüsler, J., Reiss, R.-D.: Maxima of normal random vectors: between independence and complete dependence. Stat. Probab. Lett. 7(4) 283-286 (1989)

Kabluchko, Z.: Extremes of independent Gaussian processes. To appear in Extremes. Preprint available at http://arxiv.org/abs/0909.0338 (2010)

Kabluchko, Z., Schlather, M., de Haan, L.: Stationary max-stable fields associated to negative definite functions. Ann. Probab. 37, 2042-2065 (2009)

Kallenberg, O.: Foundations of Modern Probability. Springer, New York (1997) 